\title{
Fundamental understandings of mesh process and various formats of digital data in digital dentistry
}

\author{
So-Hyoun Lee, Chang-Mo Jung, Mi-Jung Yoon, Jung-Bo Huh, Hyeonjong Lee \\ Department of Prosthodontics, School of Dentistry, Pusan National University, Yangsan, Korea
}

\begin{abstract}
Clinicians manage three-dimensional (3D) data in routine practice in the era of digital dentistry. Fundamental knowledge of digital data beyond the user's manual provided by the companies is needed to understand and handle the 3D data in clinics. The 3D data used in the clinic are acquired by scanners and processed using a step-by-step procedure. A point cloud is an initial form of raw data. The data are then filtered, and a triangle mesh is generated. The meshes could be exported in various file formats. This fundamental knowledge would be helpful for clinicians in daily practice. (JOURNAL OF DENTAL IMPLANT RESEARCH 2021;40(1):13-16)
\end{abstract}

Key Words: Digital dentistry, Mesh, Accuracy, Point cloud

\section{INTRODUCTION}

In the era of digital dentistry, intraoral scanners and computer-aided design \& manufacturing (CAD-CAM) have been widely using for treating dental patients. It helps communication between clinician and patient as well as a dental technician ${ }^{1-3)}$. The accuracy of the intraoral scanner is no longer a subject of debate compared to the conventional impression materials for a single or several units of restorations ${ }^{4-7)}$.

Clinicians obtain 3D data and manage them for various situations such as diagnosis or communication as a daily routine in the clinic. In general, clinicians would practice the use of equipment through user manuals or catalogs provided by the company. Still, fundamental knowledge regarding digital dentistry beyond the company's information would help in utilizing and understanding various digital equipment.

For example, dentists are generally learned about dental material science during their undergraduate courses. The properties of dental stone, wax, silicone, ceramic, and metal used in clinical practice are concerned as a basic knowledge for dentists. The fundamental expertise for digital dentistry should also be essential for dentists in the era of digital dentistry.

The fundamental and comprehensive information regarding scanning, data processing, and file formats were summarized in this article, which would be helpful for clinicians to understand and utilize digital sources in the clinic.

\section{PROCESS OF 3D DATA FROM SCANNING TO FINALZED FILES}

For the first step, an initial raw data Is obtained as a form of the point cloud using an intraoral scanner. The initial point cloud would have lots of noise, and the number of points would be huge since most of the intraoral scanners have around 20 30 frames per second (FPS) based on the environment. After the acquisition of the raw point cloud data, the filtering process is conducted for the second step (Fig. 1). There are various kinds of filtering algorithms such as median filter, vector median filter, adaptive mean filter, adaptive vector median filter,

Received February 25, 2021, Accepted March 5, 2021.

(c) Journal of Dental Implant Research.

This is an open access article distributed under the terms of the Creative Commons Attribution Non-Commercial License

(http://creativecommons.org/licenses/by-nc/4.0) which permits unrestricted non-commercial use, distribution, and reproduction in any medium, provided the original work is properly cited.

Correspondence to: Hyeonjong Lee, Department of Prosthodontics, School of Dentistry, Pusan National University, 20 Geumo-ro, Mulgeum-eup, Yangsan 50612, Korea.

Tel: +82-55-360-5182, Fax: +82-55-360-5134, E-mail: prostho.hjlee@pusan.ac.kr 


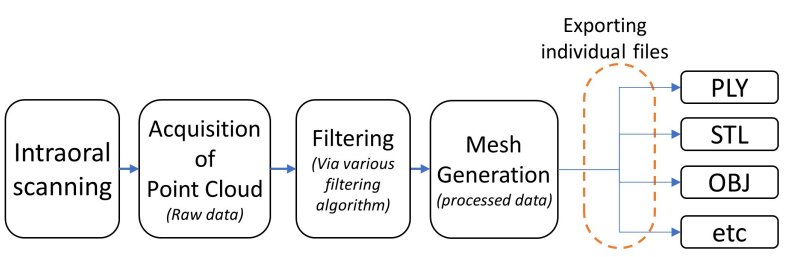

Fig. 1. Process of $3 D$ data generation from scanning to exporting files.

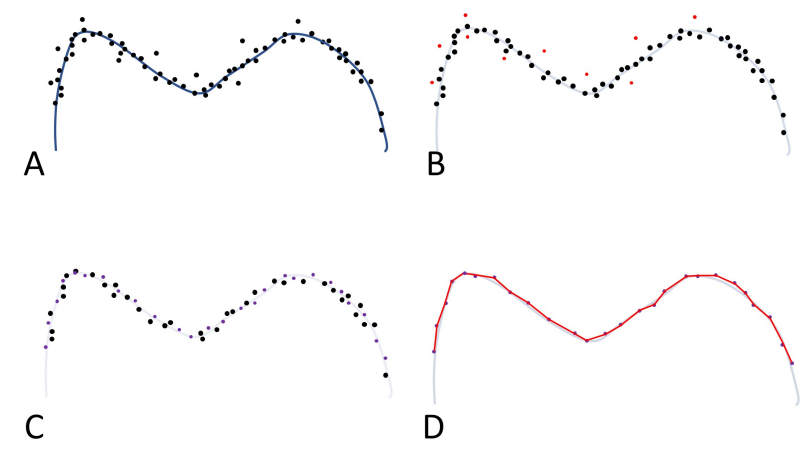

Fig. 2. (A) Acquisition of point cloud as raw data. (B) Filtering of outliers (red dots). (C) Reconstruction and selection of points depending on the setting algorithm. (D) Mesh generation.

etc. These algorithms help to erase the noise data and use the surrounding data information to fill in areas where there is insufficient data. After these algorithms, the triangle meshes are generated as a 3D surface. When the mesh is exported as individual files, it could be converted into PLY, STL, OBJ, or the other format according to the software setting.

\section{POINT CLOUD}

A point cloud is a data set that contains $x, y, z$ coordinates in 3D space. They are a group of points in the 3D space. They are an initial form or 3D data produced by lab scanners or intraoral scanners. When scanning a tooth surface, abundant point cloud data are obtained, and usually, there are scattered slightly. Outliers are also acquired during scanning, especially on the shiny surface. Since it is not a surface yet, it should be converted to the 3D surface data through the processing phase ${ }^{8}$.

\section{MESH GENERATION}

A 3D surface without flaw is required to design dental

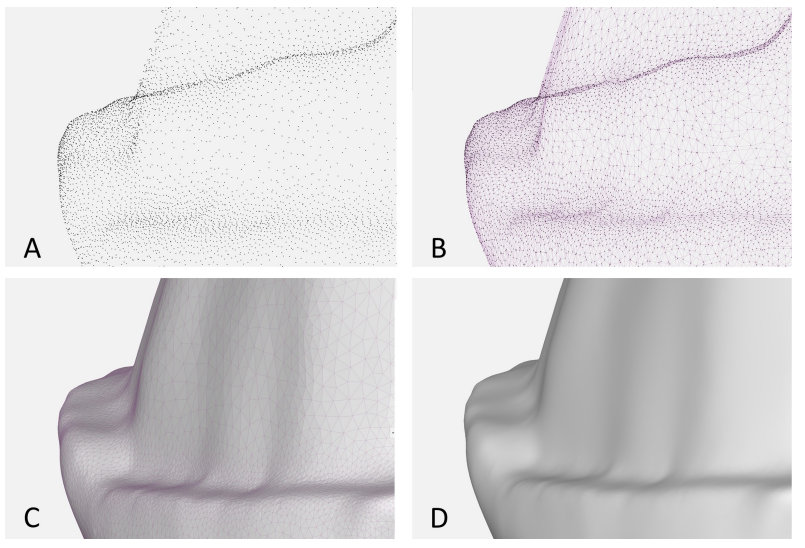

Fig. 3. Scanned data of prepared tooth for the mandibular right first molar. (A) Cloud point data. (B) Mesh generation. (C) Shading. (D) Smoothening.

restorations. Therefore, the point cloud data obtained by intraoral scanners should be converted to 3D surface data. There are various algorithms to generate a triangular mesh structure from the cloud point. The shape of the mesh could be several shapes, and a triangle shape is utilized in the dental field since it is efficient to construct an atypical surface like teeth. This mesh generation process requires lots of mathematical calculation, computing power, and memory resource. In order to effectively perform the mesh generation process, most software has been optimized to utilize the multicore process to decrease the processing time. However, this step still takes tens to hundreds of seconds depending on the performance of the computing power (Fig. 2) ${ }^{8,9)}$. The mesh generation process from an identical cloud point raw data does not ensure the same mesh generation. If the mesh generation process is performed twice using the one source of raw data, the generated two meshed would be different, and there would be a small amount of surface deviation around 1 2 micron, which would not be a problem clinically. The shortest edge of the mesh on the sharp surface, such as margins or grooves, would be around 20 50 micron, while the longest edge of the mesh on the flat buccal surface would be more than 300 $\sim 500$ micron.

\section{SHADING AND VISUALZING}

After finishing the mesh generation, the $3 \mathrm{D}$ model is colored and smoothly shaded to show an excellent visual- 
A

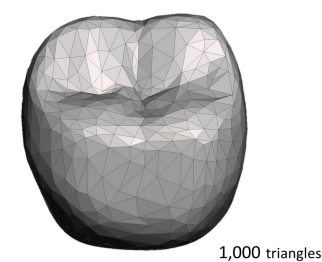

C

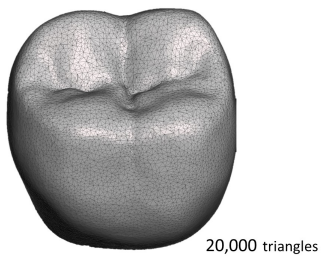

B

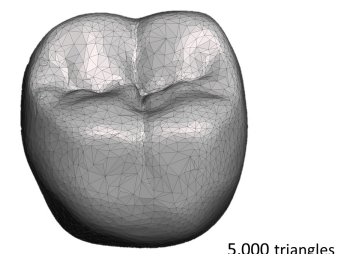

D

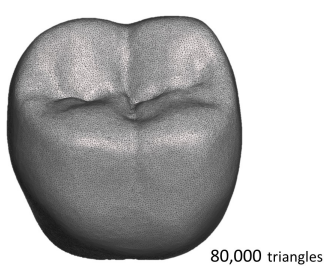

Fig. 4. Mesh generation using a different number of meshes for the mandibular first molar.

ization on the monitor (Fig. 3). This can be rotated, zoomed in and out, translated three-dimensionally. Various functions could be conducted to observe and analyze the 3D model, such as sectioning or translucency changing, measuring distances, angles, area depending on the software.

\section{THE RESOLUTTON AND ACCURACY OF DENTAL MESH DEPENDING ON THE MESH SIZE}

When teeth are scanned by an intraoral scanner, the size of the mesh can be varied depending on the resolution setting. This means that the surface of one single tooth can be composed of 5,000 triangles or even 100,000, according to the resolution setting in the software (Fig. 4). This means that the mesh size is not related to the accuracy of intraoral scanners. The size of the mesh could be tiny if an intraoral scanner is not accurate enough. Therefore, the mesh size needs to be optimized considering the accuracy of scanners, CAD software, and CAM system altogether.

\section{STEREOLITHOGRAPHY FILE FORMAT (.stl)}

STL is a file format for CAD software created by 3D Systems, and it has been widely using in the dental field ${ }^{10,11)}$. It is also called such as "Standard Tessellation Language" and "Standard Triangle Language".

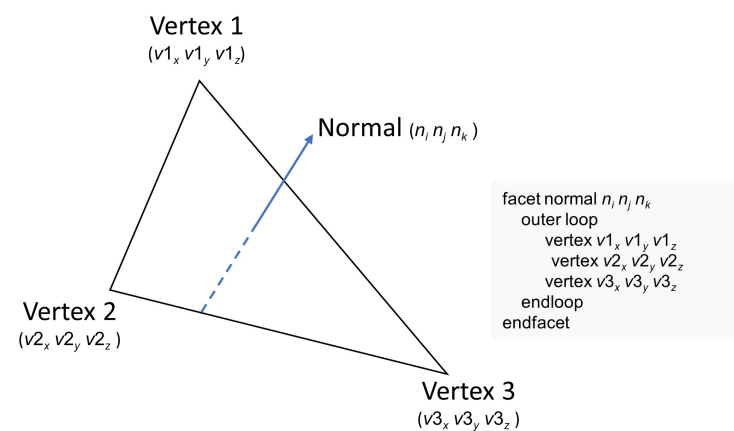

Fig. 5. A raw STL file format of a single triangle mesh composed of $3 \mathrm{D}$ data for three vertices and one normal vector.

Since it is kind of the most common format in the digital dental field, it can be exported by various dental lab scanners or intraoral scanners. A surface of a three-dimensional (3D) object is composed of a number of triangles. For example, a 3D surface of a single crown has around 20,000 50,000 triangles in STL format. Information of a single triangle occupies 50 bytes, and 20,000 triangles occupy $1 \mathrm{MB}$ of file size (Fig. 5).

A large number of triangles may increase the resolution of the mesh. However, this does not necessarily guarantee the accuracy of the restoration. From a certain level of mesh density, the accuracy of the computer-aided manufacturing does not increase, but only the file size increase due to the limitation of mechanical tolerance.

Therefore, the company sets an appropriate mesh resolution value between mesh accuracy and efficiency through file size reduction. In the future, as computing power, computational algorithms, and CAM accuracy improve, higher resolution meshes will be available in practice.

\section{WAVEFRONT FILE FORMAT (.obj)}

The OBJ is a geometry file format developed by Wavefront Technologies. This format contains not only 3D surfaces but also RGB color information. The file size is similar to the STL file. This format can be used when the information of the 3D surface with color is exported from the software as a single individual file. 


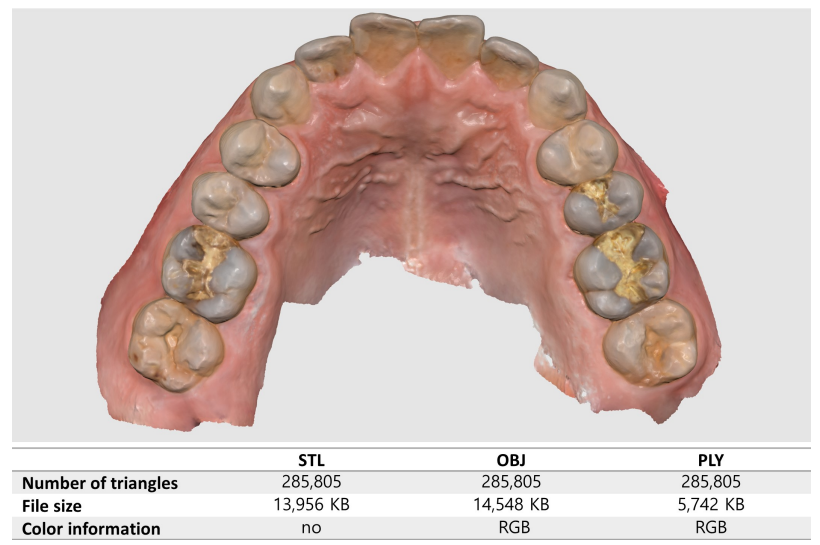

Fig. 6. Information of 3D data according to the different file format from an identical maxillary scanned data.

\section{POLYGONE FILE FORMAT (. ply)}

The PLY is another file format to store 3D data developed by Greg Turk, Stanford University. It contains 3D surface data as well as color information in RGB. The format of this file is lighter than OBJ. It can be used in one of the formats when the file is exported as an individual file from the $3 \mathrm{D}$ scanner.

\section{INFORMATION ACCURACY BASED ON THE VARIOUS FILE FORMAT}

There are no differences among STL, OBJ, or PLY in terms of three-dimensional accuracy. For example, a maxillary dentition can be scanned using an intraoral scanner, then, after mesh processing, the number of triangles and the $3 \mathrm{D}$ surface of the maxillary dentition is defined. The extension name, obj, stl, ply, means the method of writing the $3 \mathrm{D}$ information. Even the data format is different, the $3 \mathrm{D}$ data as a number does not change. Fig. 6 shows that an identical maxillary dentition is scanned then exported to three different file formats. The number of triangles and their 3D position is the same, however the file size varies. The larger file size does not guarantee three-dimensional accuracy. The STL format does not have color information, while the OBJ and PLY have color information (Fig. 6).

\section{CONCLUSION}

Clinicians need to understand the various file formats used in digital dentistry. The file size would be different according to the file format, and even they contain the same information. The mesh number should be optimized during the mesh generation process considering the accuracy of the scanner, computing power, and tolerance of the CAM system.

\section{ORCID}

Hyeonjong Lee, https://orcid.org/0000-0002-1669-2975

\section{REFERENCES}

1. Mangano Guest Editor F. Digital dentistry: the revolution has begun. Open Dent J 2018;12:59-60.

2. Silva NR, Ferencz JL, Silva GC. Expanding the application of digital dentistry with 3D patient monitoring. The International Journal of Prosthodontics 2021;34:118-9.

3. Schwendicke F. Digital dentistry: advances and challenges. J Clin Med 2020;9:4005.

4. Zarone F, Ruggiero G, Ferrari M, Mangano F, Joda T, Sorrentino R. Accuracy of a chairside intraoral scanner compared with a laboratory scanner for the completely edentulous maxilla: An in vitro 3-dimensional comparative analysis. The Journal of Prosthetic Dentistry 2020;124:761-7.

5. Rech-Ortega C, Fernandez-Estevan L, Sola-Ruiz MF, AgustinPanadero R, Labaig-Rueda C. Comparative in vitro study of the accuracy of impression techniques for dental implants: Direct technique with an elastomeric impression material versus intraoral scanner. Medicina Oral, Patologia Oral Y Cirugia Bucal. 2019;24:e89-e95.

6. Kihara $\mathrm{H}$, Hatakeyama $\mathrm{W}$, Komine $\mathrm{F}$, et al. accuracy and practicality of intraoral scanner in dentistry: A literature review. Journal of Prosthodontic Research 2020;64:109-13.

7. Camardella LT, Breuning H, de Vasconcellos Vilella O. Accuracy and reproducibility of measurements on plaster models and digital models created using an intraoral scanner. J Orofac Orthop 2017;78:211-20.

8. Zeng J, Cheung G, Ng M, Pang J, Yang C. 3D point cloud denoising using graph laplacian regularization of a low dimensional manifold model. IEEE Trans Image Process 2019.

9. Dinesh C, Cheung G, Bajic IV. Point Cloud denoising via feature graph laplacian regularization. IEEE Trans Image Process 2020;29:1413-58.

10. Afrose, Zinat. "Adaptive Methods for Point Cloud and Mesh Processing” (2018). Doctor of Philosophy (PhD), dissertation, Modeling Simul \& Visual Engineering, Old Dominion University, DOI: $10.25777 /$ ttaf-b623.

11. StereoLithography Interface Specificatino, 3D Systems, Inc., July 1988. 